\title{
The AMS-02 Silicon Tracker Status
}

\section{Matteo Duranti*}

Università degli studi di Perugia and INFN-Sezione di Perugia

Via Pascoli,

06122 Perugia, Italia

on behalf of the AMS-02 Tracker collaboration

E-mail: matteo.duranti@pg.infn.it

The Alpha Magnetic Spectrometer (AMS) space experiment is devoted to direct measurements of galactic cosmic rays (CRs) in the rigidity range $\sim \mathrm{GV}-\mathrm{TV}$. Prime goals of the AMS project are the direct search of anti-nuclei and the indirect search of dark matter particles. The final version of the experiment, AMS-02, is operating in the International Space Station (ISS) since May 2011. The AMS-02 tracking device, tout court Tracker, consists of nine planes of micro-strip silicon sensors giving a total active area of $6.2 \mathrm{~m}^{2}$. The Tracker is composed by 2264 double-sided silicon sensors $\left(72 \times 41 \mathrm{~mm}^{2}\right.$ area, $300 \mu \mathrm{m}$ thick) assembled in 192 read-out units, for a total of nearly 200,000 read-out channels. In this proceeding, we review the Tracker performance obtained with calibration and cosmic ray muon data. We also discuss the Tracker status in space after the first months of data taking.

10th International Conference on Large Scale Applications and Radiation Hardness of Semiconductor Detectors,

July 6-8, 2011

Firenze Italy

\footnotetext{
*Speaker.
} 


\section{Introduction}

The main physics objectives of the AMS project are (1) to search for primordial antimatter particles such as antihelium, (2) to search for exotic particles such as strangelets, (3) to search for dark matter particle signals through their annihilation products $\mathrm{e}^{ \pm}, \overline{\mathrm{p}}$ or $\gamma$-rays, and (4) to provide high accuracy measurements of CR spectra and chemical composition up to $Z=26$. A first version of the experiment, AMS-01, operated successfully in a 10-day shuttle mission (STS-91) in June 1998 [1]. The AMS-01 mission provided results on CR protons, helium, electrons, positrons, antiprotons, antihelium search, light nuclei and light isotopes [1, 2, 3, 4].

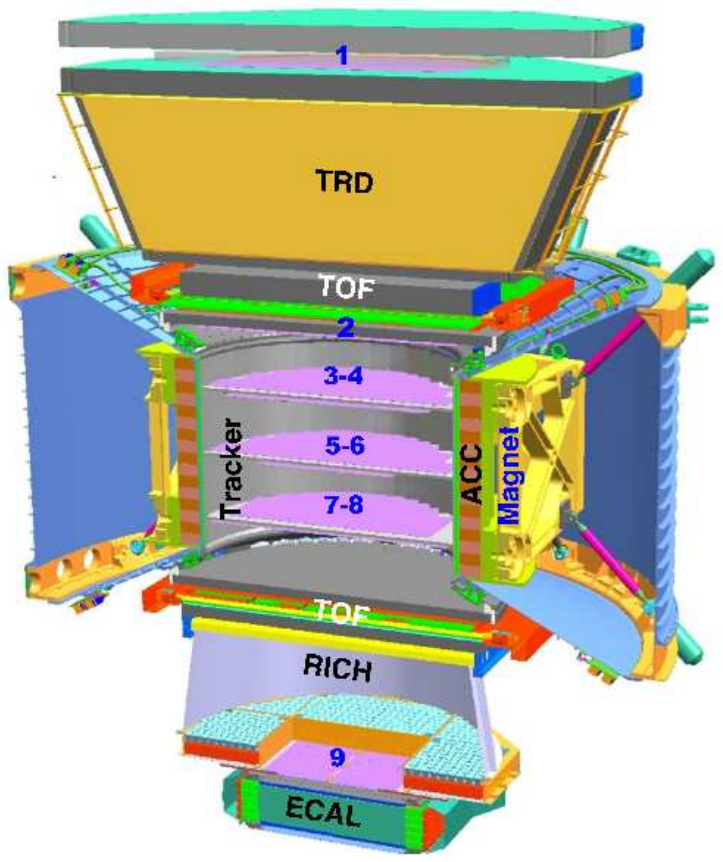

Figure 1: Schematic view of the AMS-02 spectrometer.

The second generation detector AMS-02 was launched with the STS-134 flight on May $16^{\text {th }}$ 2011 and installed in the ISS on May $19^{\text {th }} 2011$. AMS-02 is currently downlinking data at ate of $\sim 50$ million triggers per day and will be active all along the ISS lifetime. The layout of the AMS-02 detector is shown in Figure 1. It consists of a Transition Radiation Detector (TRD), a hodoscope of Time of Flight (TOF) counters, a permanent Magnet, a precision silicon Tracker, an array of Veto Counters (ACC) surrounding the Tracker, a Ring Imaging Čerenkov (RICH) detector and an Electromagnetic Calorimeter (ECAL). The programmed observation time ( $\gtrsim 10 \mathrm{yr}$ ), the large geometrical acceptance $\left(\sim 0.5 \mathrm{~m}^{2} \mathrm{sr}\right)$, the wide rigidity range $(\sim 0.2 \mathrm{GV}-\mathrm{TV})$ and the excellent identification capabilities of AMS-02 will represent a significant improvement for the flux determinations of the rare CR components and the antimatter search.

The core of the AMS-02 detector is a precision tracking device, the Tracker, which allows to determine the particle rigidity and its charge sign via the curvature radius of the particle bent trajectories [5]. Multiple energy loss measurements in the silicon layers allows to determine the 
absolute charge of the particles [6]. Below we provide a basic description of the Tracker and discuss its performance and functionality with ground muon data and flight CR data.

\section{The AMS-02 Silicon Tracker}

The Tracker is composed of 2264 double-sided silicon micro-strip sensors of area $72 \times 41 \mathrm{~mm}^{2}$ and thickness $300 \mu \mathrm{m}$. These sensors are arranged into 192 independent units, the ladders, which are assembled in nine layers as schematically shown in Figure 1. Seven layers are placed inside the magnet bore on four support planes, while two external layers are installed on both sides of the spectrometer, above the TRD system and between RICH and ECAL respectively. The support planes are made of an aluminum honeycomb structure enclosed within thin carbon fiber skins. On opposite sides of each silicon sensor, $\mathrm{p}^{+}$(junction) and $\mathrm{n}^{+}$(ohmic) strips are implanted along orthogonal directions with implantation (readout) pitches of 27.5 (110) $\mu \mathrm{m}$ and 104 (208) $\mu \mathrm{m}$ respectively. The p-side strips provide the measurement of the bending coordinate in the spectrometer. The ladder design and its components are sketched in Figure 2. Running along the ladder length, the strips from different sensors are daisy chained up to the ladder end where they are connected to a hybrid, which provides bias voltages and front-end readout chips [7]. The n-side strips, providing the measurement of the non-bending coordinate, are also routed to the ladder end by means of a kapton cable which distributes the signals from alternating sensors to the same readout channel. The 640(384) readout strips from the $\mathrm{p}(\mathrm{n})$ sides of each ladder are AC-coupled to a 64 channel

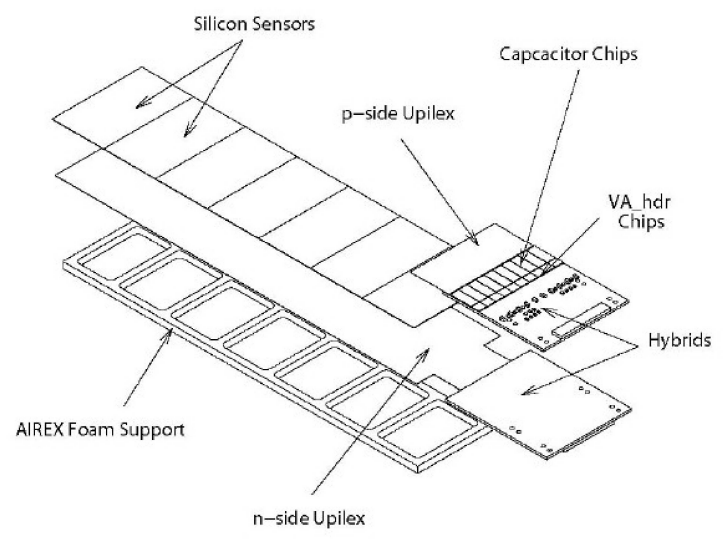

Figure 2: The components of a AMS-02 silicon tracker ladder.

low-noise, high dynamic range readout chip VA_hdr 9 a via $700 \mathrm{pF}$ capacitors. The analog signals of each hybrid pair, for $\mathrm{p}$ and $\mathrm{n}$ side, are transferred to a Tracker Data Reduction (TDR) board which is equipped with three 12-bit ADCs, Field Programmable Gate Arrays (FPGAs) and Digital Signal Processors (DSPs) for data reduction. The ADC information on the readout strips include a pedestal, a common noise, a single-strip noise and an eventual signal. The mean and root mean square (RMS) of the pedestal are determined in a calibration run at the beginning of each scientific run. A valid signal is defined by a threshold applied to the signal-to-noise ratio for each readout strip after pedestal and common noise subtraction. The noise refers to the RMS value obtained in the calibration run. 


\section{Tracker Performance and Status}

Before the AMS-02 launch, the whole detector has been extensively calibrated with proton beams at CERN, Geneva, and with CR muons at the Kennedy Space Center (KSC) facilities, Cape Canaveral. The pre-flight detector performance were thoroughly understood. Figure 3 shows the Tracker alignment accuracy obtained with the CR muon data collected at KSC. The on-ground alignment of the external Tracker layers can be achieved with an accuracy of $2 \mu \mathrm{m}(1 \mu \mathrm{m}$ for the seven inner silicon layers) by means of CR muons in a time interval of $10^{3} \mathrm{~min}$, which ensures the design spatial resolution of the Tracker in the bending plane of $10 \mu \mathrm{m}$. The right panel of
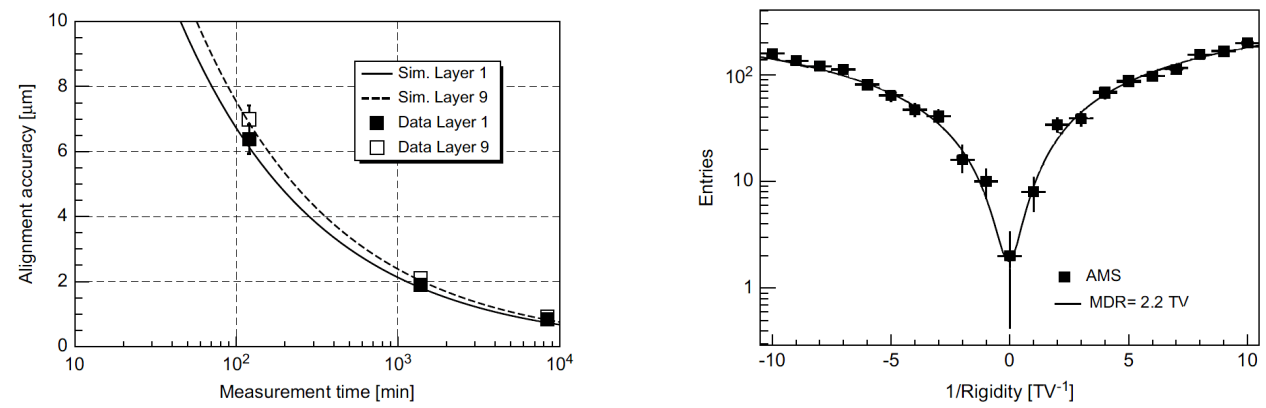

Figure 3: Left: Tracker alignment accuracy measured with CR muons at KSC. Right: CR measurement of the $1 / R$ distribution after Tracker alignment.

the figure shows the signed $1 / R$ distribution of CR muons $\left(\mu^{+}\right.$and $\left.\mu^{-}\right)$after the whole Tracker alignment was performed [8]. It can be seen that the minimum at $1 / R=0$ corresponds to the Monte-Carlo expectations for a maximum detectable rigidity (MDR) larger than $2 \mathrm{TeV}$, where the MDR is defined as the rigidity value for which the resolution $\Delta R / R$ approaches the unity [9].

Since its activation on May $19^{\text {th }} 2011$, the AMS-02 detector is working nominally onboard the ISS and no issues were noticed. The Tracker performance and its functionality are continuously monitored at the Payload Operation Control Center (POCC) located at CERN. Calibration runs are performed every 46 minutes, when the ISS is passing above the equator, measuring pedestal and noise level of each channel and assigning a status bit to each of them to avoid to use noisy or dead strips in the science data analysis. The Tracker calibration response is stable in time, as seen from the monitoring of the pedestal and noise levels. Figure 4 shows the average noise the p-side as function of time for all the nine layers. The Tracker is also characterized by a uniform ladder response, which is monitored by the measurement of signals corresponding to proton and helium charges for each ladder. The detector response to different charges is shown in Figure 5. In the figure, the square root of the mean amplitude of the Tracker signals is used as a charge deposition estimator (CE). The CE is plotted as a function of the measured rigidity, between 0.1 and $200 \mathrm{GV}$, using the data collected in 40 ISS orbits around the Earth that correspond to 60 hours of data taking time. The different CR species such as electrons, protons, helium nuclei (from bottom to top) as well as the less abundant $Z>2$ nuclei are apparent from the figure. The figure also shows the low rigidity behavior and the $Z$ dependence of the energy depositions, according the $\sim Z^{2} / \beta$ BetheBloch dependence and consistently with previous performance studies [6, 3]. Intensive studies are currently ongoing for modeling the detector response to the different charges and performing the 


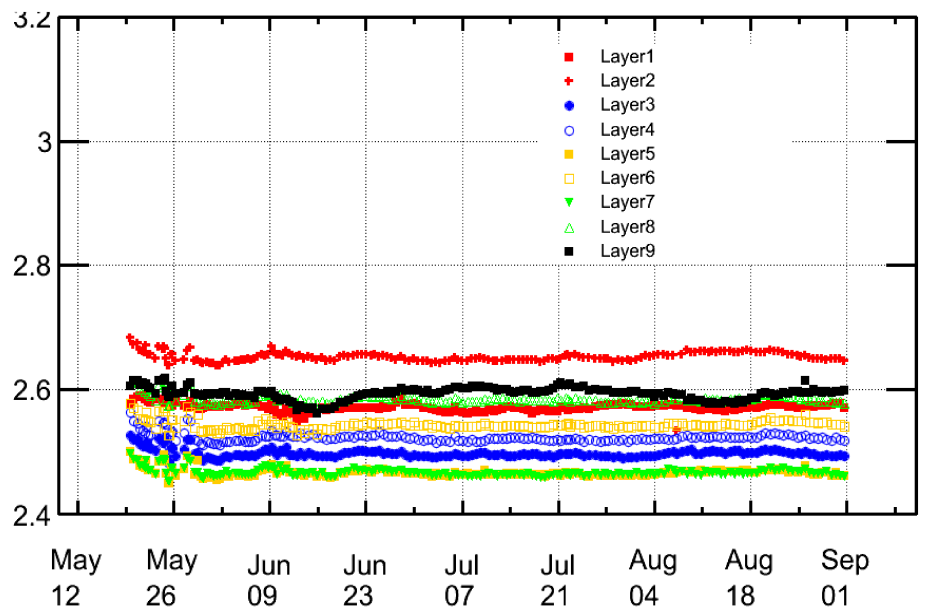

Figure 4: Average noise measured on the p-side, for all of the layers, as a function of time (ADC units).

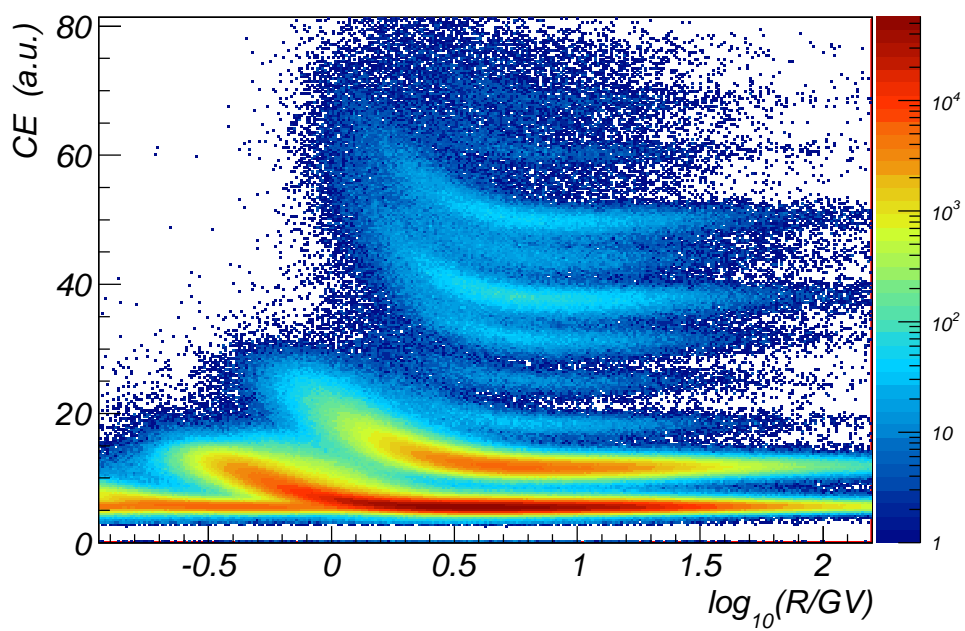

Figure 5: Tracker charge estimator as a function of the rigidity after 60 hours of data taking time.

in-flight alignment with CR data.

\section{Conclusions}

AMS-02 is collecting data since May $19^{\text {th }}$ at a rate of $\sim 50$ million triggers per day. All the sub-detectors and the data acquisition system are working nominally. The Tracker is exhibiting the expected behavior with high stability of pedestal and noise levels. Furthermore, it has been observed a high ladder uniformity in their efficiency response to CR particles. The Tracker can reach a rigidity resolution of $\sim 10 \%$ at $10 \mathrm{GV}$ and has a MDR of about $2 \mathrm{TV}$ for $Z=1$ particles. The energy depositions, combined with the signals from RICH and TOF allow the charge determination from $Z=1$ to $Z=26$. Intensive data analysis activities are being carried on to understand all 
the detector systematics, to perform the Tracker alignment and to model its response to different charges. All these aspects are crucial to exploit the full AMS-02 detector potentiality.

\section{Acknowledgements}

The author wishes to thank the colleagues in AMS-02 and in particular N. Tomassetti for the careful reading of the manuscript.

This work was partly funded by the Agenzia Spaziale Italiana (ASI Contract 075/09, AMS-02-Fase E2 ed analisi dati).

\section{References}

[1] J. Alcaraz et al., The Alpha Magnetic Spectrometer (AMS) on the International Space Station: Part I results from the test flight on the space shuttle, Physics Reports, 366 (2002) 331-405.

[2] M. Aguilar et al., Cosmic-ray positron fraction measurement from 1 to $30 \mathrm{GeV}$ with AMS-01, Physics Letters B, 646 (2007) 145-154.

[3] M. Aguilar et al., Relative composition and energy spectra of light nuclei in cosmic rays: results from AMS-01, The Astrophysical Journal, 724 (2010) 329-340.

[4] M. Aguilar et al., Isotopic composition of light nuclei in cosmic rays: results from AMS-01, The Astrophysical Journal, 736 (2011) 105.

[5] J. Alcaraz et al., The Alpha Magnetic Spectrometer Silicon Tracker: Performance results with Protons and Helium nuclei, Nuclear Instruments and Methods A, 593 (2008) 376-398.

[6] B. Alpat et al., Charge determination of nuclei with the AMS-02 silicon tracker, Nuclear Instruments and Methods A, 518 (2004) 121-130.

[7] B. Alpat et al., High-precision tracking and charge selection with silicon strip detectors for relativistic ions, Nuclear Instruments and Methods A, 446 (2000) 552.

[8] K. Lübelsmeyer et al., Upgrade of the Alpha Magnetic Spectrometer (AMS-02) for long term operation on the International Space Station (ISS), Nuclear Instruments and Methods A, 654 (1) (2011) 639-648.

[9] B. Alpat et al. The internal alignment and position resolution of the AMS-02 silicon tracker determined with cosmic-ray muons, Nuclear Instruments and Methods A, 613 (2010) 207-217. 